\title{
A MONTE CARLO STUDY ON THE DYNAMICAL FLUCTUATIONS INSIDE QUARK-GLUON FUSION PRODUCTS IN QCD-INSTANTON INDUCED PROCESSES IN DEEP-INELASTIC SCATTERING
}

\author{
ZHANG KUNSHI \\ School of Physical Science and Technology, Yangtze University, \\ Jingzhou 434023, China \\ zhangks@iopp.ccnu.edu.cn \\ B. B. LEVCHENKO* \\ Institute of Nuclear Physics, Moscow State University, \\ RU-119992 Moscow, Russia \\ levtchen@mail.desy.de \\ WEI FEI, LE TIAN, HU XIONGJUN, XU MINGMEI and LIU LIANSHOU ${ }^{\dagger}$ \\ Institute of Particle Physics, Huazhong Normal University, \\ Wuhan 430079, China \\ †liuls@iopp.ccnu.edu.cn
}

Received 7 February 2007

\begin{abstract}
The dynamical fluctuations in instanton induced events in $e^{+} p$ deep-inelastic scattering are studied using QCDINS 2.0 Monte Carlo generator. The hadron system produced from the fusion of quark-gluon in the presence of instanton, referred to as instanton final state (IFS), is identified by a $p_{z}$-sorting together with a $\theta_{\text {cut }}$ method. The dynamical fluctuations inside the current jet and IFS are compared by means of the method of normalized factorial moments. The current jets are found to possess the typical characteristics of a "circular jet" with axial symmetry, while the dynamical fluctuations inside the IFS represent a three-dimensional isotropy.
\end{abstract}

Keywords: Instanton; quark-gluon fusion; deep-inelastic scattering; dynamical fluctuation.

\section{Introduction}

In a non-Abelian gauge theory such as quantum chromodynamics (QCD) the vacuum has a complicated structure. There are nonperturbative fluctuations of the

*Currently at DESY (ZEUS), Hamburg. 
gauge fields, describing tunneling transition between the degenerate ground states (degenerate vacua) of different topology, called instanton. The instantons and antiinstantons carry integer topological charges $Q=1$ and -1 , respectively, while the usual perturbation theory exists in the sector $Q=0$.

Deep-inelastic scattering (DIS) accompanied by instanton-induced hard processes, cannot be described by conventional perturbation theory. The chirality conservation is violated in case of strong (QCD) instantons (antiinstantons). A study of DIS events induced by instantons is of fundamental significance for a thorough understanding of the basic theory of strong interaction - QCD. ${ }^{1,2}$

The $e P$ deep-inelastic scattering has been proved to be an appropriate laboratory to discover and study the instanton-induced events (I-events). The I-events in DIS arise predominantly from quark-gluon fusion in an instanton background. In such a process, a virtual photon splits into a $q \bar{q}$-pair, one of which, referred to as current quark, fragments directly, forming a current jet, while the other one fuses with a gluon from the proton in the background of an instanton (I) or an antiinstanton $(\overline{\mathrm{I}})$. The latter forms a cluster of $\left(2 n_{f}-1\right) q+n_{g} g$ partons, where $n_{f}$ is the number of quark flavors that are kinematically allowed in the experiment, and $n_{g}$ is the number of gluons, and then fragments into final state through hadronization with high multiplicity and high transverse energy. In the following we will refer to the quark-gluon fusion product in the presence of an instanton as the instanton final state, or IFS for short.

Although the hard I-events can be calculated within instanton-perturbation theory ${ }^{1}$ and the two experimental groups, $\mathrm{H}^{3}{ }^{3}$ and $\mathrm{ZEUS},{ }^{4}$ of DESY-HERA in Germany have been actively doing experimental searches for instanton-induced events, the experimental verifications of the instanton theory set only upper limits on the cross-section of such processes. At present, together with the experimental searches for the instanton-induced events, a Monte Carlo study on the physical properties of IFS is also important.

The QCDINS 2.0 Monte Carlo event generator ${ }^{5}$ provides us a useful tool to study the properties of instanton-induced hard processes. The aim of the present paper is to use this Monte Carlo generator to study the dynamical fluctuations inside the instanton final state (IFS) in comparison with those inside the current jet. Through this study the symmetry property of these systems with respect to dynamical fluctuations will be explored. In particular, the expected isotropy of IFS will be confirmed and the current jet as a circular or visible jet will be illustrated.

\section{Event Samples of Instanton Final State and Current Jet}

The Monte Carlo event generator QCDINS 2.0 is designed to simulate the QCDinstanton induced processes in deep-inelastic $e P$ scattering. It is based on the instanton perturbation theory and embedded in the general hadronic event simulation package HERWIG. ${ }^{6}$ 
In our Monte Carlo simulation, $e^{+} p$ deep-inelastic scattering events are produced by QCDINS 2.0 with the proton and electron energies equal to 820 and $27.5 \mathrm{GeV}$, respectively. The scattered electron and the proton remnant are discarded at the beginning. Then the $p_{z}$ sorting $^{7}$ together with a $\theta_{\text {cut }}$ method is applied in the common center-of-mass system (CM2) of the current jet and instanton final state to distinguish them apart.

This method is to take the $+z$ axis along the current quark direction. Then order all the $n$ particles in the CM2 system according to their $p_{z}$ values and renumber them so that $p_{z 1}>p_{z 2}>\cdots>p_{z n}$. The bigger the $p_{z}$ of a particle the more likely this particle to be in the current jet. Starting from the number one particle, sum up the particle energy $\varepsilon_{i}(i=1,2, \ldots, k, \ldots, n)$ up to the $k$ th one to make the energy of the reconstructed current jet $E_{\text {jet }}=\sum_{i=1}^{k} \varepsilon_{i}$ most close to the current-quark energy $E_{C}$ before hadronization. At the same time sum up the remaining particles in the CM2 system, making the energy of the reconstructed instanton final state $E_{\text {ins }}=\sum_{i=k+1}^{n} \varepsilon_{i}$ most close to the "instanton energy" $E_{\mathrm{I}}$. The latter is equal to the sum of the energies of the fusing quark and gluon before fragmentation. Then the particles from the first one to the $k$ th one are classified to be the hadrons in the current jet and the particles from the $(k+1)$ th to the $n$th belong to the products of instanton (IFS). Correspondingly, the relative error of the reconstructed current jet and IFS energies are $\Delta E_{\text {jet }}$ and $\Delta E_{\mathrm{I}}$, respectively. The relative error of a quantity $X$ is defined as $\Delta X=\frac{X_{\mathrm{rec}}-X}{X}$, where $X$ is the real value in parton level and $X_{\mathrm{rec}}$ is the reconstructed value. Then choose an appropriate number $k$ to make the error of the total reconstructed energy $\Delta E=0.4 \times\left|\Delta E_{\text {jet }}\right|+0.6 \times\left|\Delta E_{\mathrm{I}}\right|$ minimum. Finally, an energy cut is added to make the events remained at last satisfy $\Delta E<10 \%$, and a $\theta_{\text {cut }}=40^{\circ}$ is chosen, so that if the polar angle $\theta$ of any particle in the current jet relative to the jet axis is greater than $\theta_{\text {cut }}$, then throw away this event. The distributions of the reconstruction errors $\Delta E_{\text {jet }}$ for the jet energy, $\Delta E_{\mathrm{I}}$ for the instanton energy and $\Delta M_{\mathrm{I}}$ for the instanton mass in this method are shown in Fig. 1.

In our Monte Carlo simulation a total number of 1200000 events of $e^{+} p$ deepinelastic scattering are produced by QCDINS 2.0 generator and 119328 events of the current jet and the instanton final state are obtained after passing all cuts and are used in the following analysis.

\section{Dynamical Fluctuations inside IFS and Current Jet}

To study the dynamical fluctuations inside the final state particles of a multiparticle system produced in high energy collision is an effective way for exploring the internal property of the system and the dynamics of strong interaction.

Since the factorial moments (FM) were proposed to be used in the study of nonstatistical fluctuations (or dynamical fluctuations) in multiparticle production by Białas and Peschanski, ${ }^{8}$ a large number of efforts have been made to search for the anomalous scaling of $F_{q}(M)$ in experiments,

$$
F_{q}(M) \propto M^{-\phi} \quad(M \rightarrow \infty) .
$$



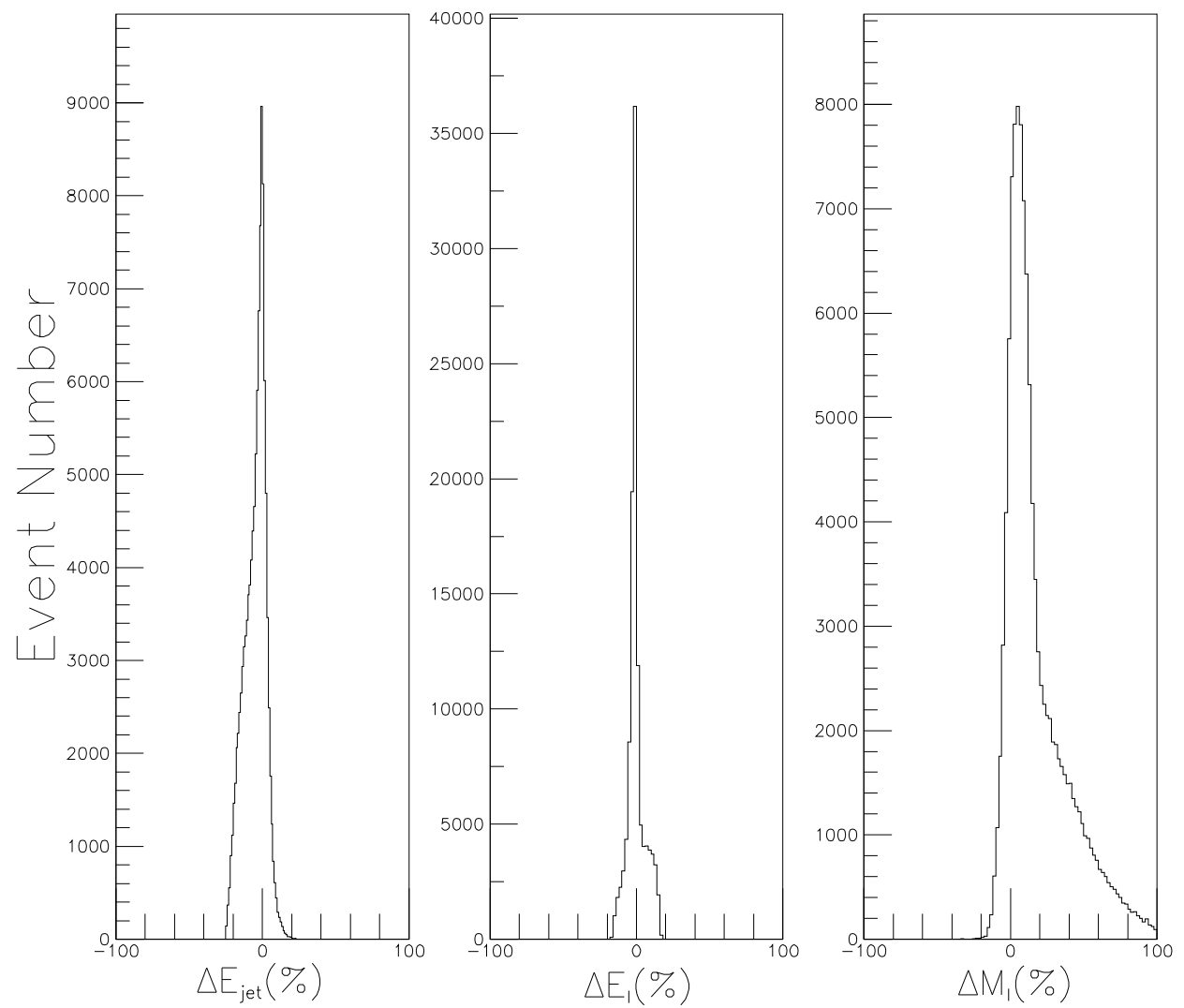

Fig. 1. The distributions of the relative reconstruction-error $\Delta E_{\text {jet }}$ for jet energy, $\Delta E_{\mathrm{I}}$ for instanton energy and $\Delta M_{\mathrm{I}}$ for instanton mass in $p_{z}$-sorting method with energy- and $\theta$-cuts.

The results showed that the one-dimensional factorial moments do not have such a power law behavior with the diminishing of phase scale, but tend to go to saturation instead. As Ochs pointed out,${ }^{9}$ the anomalous scaling of dynamical fluctuations occurring in higher-dimensional (2D or $3 \mathrm{D})$ phase space have a projection effect on the fluctuations in lower-dimensional space causing the second-order 1D factorial moments $F_{2}^{(a)}\left(M_{a}\right)$ go to saturation according to the rule

$$
F_{2}^{(a)}\left(M_{a}\right)=A_{a}-B_{a} M_{a}^{-\gamma_{a}}
$$

where $a=1,2,3$ denote the $1 \mathrm{D}$ variables $p_{x}, p_{y}, p_{z}$ or $y, p_{t}, \varphi$, respectively. $A_{a}, B_{a}$ and $\gamma_{a}$ are constants and $M_{a}$ is the total number of partition of a given variable.

The nature of the dynamical fluctuations can be expressed in terms of the Hurst exponent $H_{a b},{ }^{11}$ which can be obtained from the values of $\gamma_{a}$ and $\gamma_{b}$ as

$$
H_{a b}=\frac{1+\gamma_{b}}{1+\gamma_{a}}
$$


The dynamical fluctuations are isotropic (self-similar fractal) when $H_{a b}=1$, and anisotropic (self-affine fractal) when $H_{a b} \neq 1 .^{10}$

We first try to answer the question, whether the dynamical fluctuations of IFS and current jet is isotropic in their own rest frames. For this purpose we use the Cartesian coordinates $p_{x}, p_{y}, p_{z}$ as the three variables both for the IFS and for the current jet in their own c.m. frames, respectively. The phase space region $-3<p_{x}<$ $3,-3<p_{y}<3,-3<p_{z}<3 \mathrm{GeV} / c$ are divided isotropically. In order to avoid the influence of a nonflat distribution of the variables on the investigation of the dynamical fluctuations, all the variables are transformed into their corresponding cumulant form, ${ }^{12}$

$$
X\left(p_{i}\right)=\frac{\int_{p_{i a}}^{p_{i}} \rho\left(p_{i}\right) d p_{i}}{\int_{p_{i a}}^{p_{i b}} \rho\left(p_{i}\right) d p_{i}} \quad(i=x, y, z) .
$$

In the following the factorial moments $F_{q}\left(X\left(p_{i}\right)\right)$ are always calculated instead of $F_{q}\left(p_{i}\right)$. However, to simplify the notation we will still use the latter $-F_{q}\left(p_{i}\right)$ to denote the former $-F_{q}\left(X\left(p_{i}\right)\right)$.

The distributions of the three cumulant variables $X\left(p_{x}\right), X\left(p_{y}\right), X\left(p_{z}\right)$ become flat in the region $[0,1]$, except at the end point. As example, the distributions of the momentum components $p_{x}, p_{y}, p_{z}$ and their corresponding cumulant variables $X\left(p_{x}\right), X\left(p_{y}\right), X\left(p_{z}\right)$ of IFS are shown in Fig. 2 upper and lower panels, respectively.
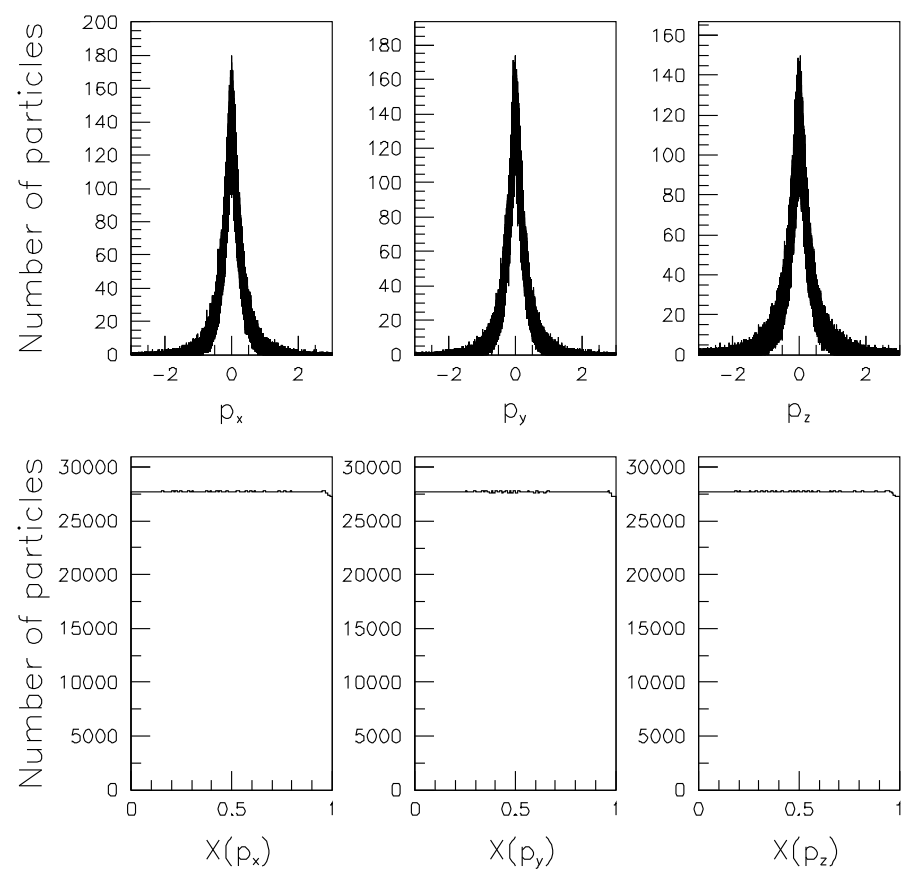

Fig. 2. The distributions of the momentum components (upper panel) and their corresponding cumulant variables (lower panel) of the IFS. 


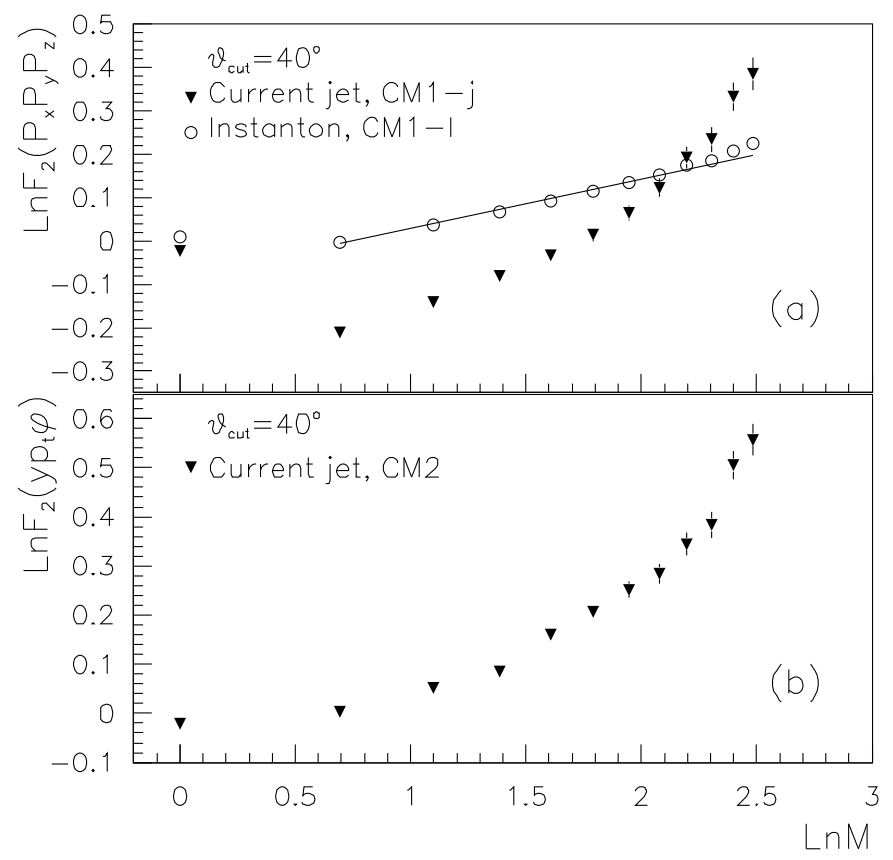

Fig. 3. (a) The $\ln F_{2}\left(p_{x}, p_{y}, p_{z}\right) \sim \ln M$ plots of the instanton final state and the current jet in their own rest frames, (b) that of the current jet in the CM2 frame.

Then the second-order 1D and 3D factorial moments $F_{2}\left(p_{x}\right), F_{2}\left(p_{y}\right), F_{2}\left(p_{z}\right)$ and $F_{2}\left(p_{x}, p_{y}, p_{z}\right)$ are calculated. The results for $\ln F_{2}\left(p_{x}, p_{y}, p_{z}\right)$ vs $\ln M$ are shown in Fig. 3(a). It can be seen that the $\ln F_{2}\left(p_{x}, p_{y}, p_{z}\right)$ vs $\ln M$ for instanton final state is a straight line after omitting the first point to eliminate the influence of momentum conservation, ${ }^{13}$ while that of the current jet, shown as downward triangles in Fig. 3(a), is an upward-bending curve. These results show that the factorial moment $F_{2}$ of IFS obeys the anomalous scaling law Eq. (2) when the 3D phase space is partitioned equally in the three dimensions. This means that the dynamical fluctuations inside IFS is 3-dimensionally isotropic in its rest frame. On the contrary, the $F_{2}$ of current jet does not obey the scaling law, showing that the dynamical fluctuations inside jet is anisotropic.

The anisotropy of the dynamical fluctuations inside jet is natural because jet is the fragmentation product of a parton. This is evident in the CM2 frame - the common center of mass frame of the current jet and the instanton final state. In this frame the jet has axial symmetry around the jet-momentum. However, even in the jet-CM1 frame, i.e. the rest frame of jet alone, there is also axial symmetry, because the boosting from CM2 to CM1, which is along the jet axis, does not change the momenta in transverse plane while the momenta along the jet axis are divided into two opposite directions with half value in each direction. Thus the two directions along the jet axis is still privileged in this frame. 
The downward solid triangles in Fig. 3(a) show the anisotropy of dynamical fluctuations inside jet in the jet-CM1 frame using the momenta $p_{i}, i=x, y, z$ as variables. A more natural way for checking this anisotropy is to work in the CM2 frame using the rapidity $y$, transverse momentum $p_{t}$ and azimuthal $\varphi$ of particles inside jet as variables. In defining these variables the direction of currentjet momentum is chosen as longitudinal.

The second-order factorial moments of the new variables $F_{2}(y), F_{2}\left(p_{t}\right), F_{2}(\varphi)$ and $F_{2}\left(y, p_{t}, \varphi\right)$ are calculated again and the $\ln F_{2}\left(y, p_{t}, \varphi\right) \sim \ln M$ for current jet is plotted in Fig. 3(b). Comparing the two curves for current jet in Figs. 3(a) and 3 (b), it can be seen that although the coordinate frame and variables have been changed, the upward-bending tendency remains in common, showing the robust nature of the anisotropy of dynamical fluctuations inside jets.

The $\ln F_{2}$ vs $\ln M$ plots in Fig. 3(a) show clearly the difference in the dynamical fluctuations inside instanton final state and current jet. In order to be in more detail, a fitting to the saturation formula, Eq. (2), of the second order 1D factorial moments $F_{2}(i)\left(i=p_{x}, p_{y}, p_{z}\right.$ for instanton and $y, p_{t}, \varphi$ for current jet) vs the partition number $M=1,2, \ldots, 40$ is carried out and the corresponding parameters $\gamma_{i}\left(\gamma_{p_{x}}\right.$, $\gamma_{p_{y}}, \gamma_{p_{z}}$ for instanton and $\gamma_{y}, \gamma_{p_{t}}, \gamma_{\varphi}$ for current jet) are obtained, respectively. To eliminate the influence of momentum conservation, ${ }^{13}$ the first point (first three points in case of $F_{2}(\varphi)$ ) are omitted when fitting the data to Eq. (2). The fitting results are listed in Table 1. Accordingly, the Hurst exponents of the instanton final state and current jet deduced from Table 1 and Eq. (3) are listed in Table 2.

\section{Analysis and Discussions}

Since the products of the final state of QCD-instanton induced event in $e^{+} p$ deepinelastic scattering should be neutral in color, it is natural that there is color correlation among the current jet, the instanton final state and the proton remnant, i.e. color exchange must take place among the partons of these three parts.

Table 1. The saturation exponents $\gamma_{i}$ of $1 \mathrm{D}$ second-order factorial moments.

\begin{tabular}{rrr|rrr}
\hline \multicolumn{3}{c|}{ Instanton final state } & \multicolumn{3}{c}{ Current jet } \\
$\gamma_{p_{x}}$ & $\gamma_{p_{y}}$ & $\gamma_{p_{z}}$ & $\gamma_{y}$ & $\gamma_{p_{t}}$ & \multicolumn{1}{c}{$\gamma_{\varphi}$} \\
\hline 0.024 & 0.019 & 0.220 & 0.768 & 0.011 & 0.014 \\
\pm 0.003 & \pm 0.002 & \pm 0.015 & \pm 0.123 & \pm 0.001 & \pm 0.001 \\
\hline
\end{tabular}

Table 2. The Hurst exponents of instanton final states and current jet.

\begin{tabular}{rrr|rrr}
\hline \multicolumn{3}{c|}{ Instanton final state } & \multicolumn{3}{c}{ Current jet } \\
$H_{p_{x} p_{y}}$ & $H_{p_{x} p_{z}}$ & $H_{p_{y} p_{z}}$ & $H_{y p_{t}}$ & $H_{y \varphi}$ & $H_{p_{t} \varphi}$ \\
\hline 0.996 & 1.192 & 1.197 & 0.572 & 0.573 & 1.002 \\
\pm 0.004 & \pm 0.018 & \pm 0.017 & \pm 0.040 & \pm 0.040 & \pm 0.001 \\
\hline
\end{tabular}


Therefore, the above-mentioned three subsystems are not completely independent. When multiplicities are not very low the color correlation among the three systems can be neglected in the first approximation. In the present paper we have taken this approximation.

The $\log -\log$ plot of $3 \mathrm{D}$ factorial moments vs partition number $M$, shown in Fig. 3(a), turns out to be a good straight line for IFS and bending upward for current jet, i.e. the former obeys the anomalous scaling law Eq. (1) very well, while the latter does not. This indicates that the dynamical fluctuations in the IFS are isotropic while that in the current jet are anisotropic. The isotropy of dynamical fluctuations inside the IFS is an indication for the local equilibrium in this system. ${ }^{14}$

The Hurst exponents listed in Table 2 represent the characteristic property:

$$
\begin{aligned}
& H_{p_{x} p_{y}} \simeq H_{p_{x} p_{z}} \simeq H_{p_{y} p_{z}} \simeq 1 \quad \text { for IFS }, \\
& H_{y p_{t}} \simeq H_{y \varphi}<H_{p_{t} \varphi} \simeq 1 \quad \text { for current jet } .
\end{aligned}
$$

This feature, besides indicating the isotropy of the dynamical fluctuations in the IFS and the anisotropy in the current jet, shows further that the dynamical fluctuations inside current jets are circular in the transverse plane.

In our method for identifying IFS and current jet a cut $\theta_{\text {cut }}=40^{\circ}$ has been added on the jet cone size. In order to study the influence of the value of $\theta_{\text {cut }}$ on the results obtained, the above analysis has been repeated for three other values of $\theta_{\text {cut }}$. The results listed in Tables 3 and 4 show that although the values of Hurst exponents change a little for different values of $\theta_{\text {cut }}$, the regularity shown in Eqs. (5) and (6) always hold.

Table 3. The Hurst exponents of the instanton final state corresponding to four different values of $\theta_{\text {cut }}$.

\begin{tabular}{l|ccc}
\hline \multirow{2}{*}{$\theta_{\text {cut }}$} & \multicolumn{3}{|c}{ Instanton final state } \\
\cline { 2 - 4 } & $H_{p_{x} p_{y}}$ & $H_{p_{x} p_{z}}$ & $H_{p_{y} p_{z}}$ \\
\hline $30^{\circ}$ & $0.994 \pm 0.005$ & $1.226 \pm 0.018$ & $1.234 \pm 0.017$ \\
$40^{\circ}$ & $0.996 \pm 0.004$ & $1.192 \pm 0.018$ & $1.197 \pm 0.017$ \\
$50^{\circ}$ & $1.000 \pm 0.004$ & $1.130 \pm 0.011$ & $1.130 \pm 0.012$ \\
$60^{\circ}$ & $1.006 \pm 0.005$ & $1.113 \pm 0.006$ & $1.106 \pm 0.007$ \\
\hline
\end{tabular}

Table 4. The Hurst exponents of the current jet corresponding to four different values of $\theta_{\text {cut }}$.

\begin{tabular}{l|ccc}
\hline \multirow{2}{*}{$\theta_{\text {cut }}$} & \multicolumn{3}{|c}{ Current jet } \\
\cline { 2 - 4 } & $H_{y p_{t}}$ & $H_{y \varphi}$ & $H_{p_{t} \varphi}$ \\
\hline $30^{\circ}$ & $0.627 \pm 0.032$ & $0.629 \pm 0.033$ & $1.003 \pm 0.001$ \\
$40^{\circ}$ & $0.572 \pm 0.040$ & $0.573 \pm 0.040$ & $1.002 \pm 0.001$ \\
$50^{\circ}$ & $0.510 \pm 0.034$ & $0.510 \pm 0.034$ & $0.999 \pm 0.001$ \\
$60^{\circ}$ & $0.465 \pm 0.028$ & $0.469 \pm 0.029$ & $1.009 \pm 0.002$ \\
\hline
\end{tabular}


It has been shown in Refs. 15-18 that the circular jets with respect to dynamical fluctuations are consistent with the visible jets in high energy collision experiments. The results of the present paper on the dynamical fluctuations inside current jets indicate that the current jets possess the typical characteristics of circular jets with respect to dynamical fluctuations. This means that the current jets in the instanton-induced events are visible jets in high energy $e^{+} p$ deep-inelastic scattering experiments.

\section{Acknowledgments}

This work is supported by the NSFC-RFBR cooperative research project 10475030 and 05-02-39028-NNSF-a, the NSFC projects 10375025, 90503001, the Cultivation Fund of the Key Scientific and the Technical Innovation Project, Ministry of Education of China No. CFKSTIP-704035 and by the Team Project of Sci-Tech Innovation for Excellent Young and Middle-Aged University Teachers of Hubei Province (2003-16).

The authors are grateful to Chen Gang for helpful suggestions.

\section{References}

1. S. Moch, A. Ringwald and F. Schrempp, Nucl. Phys. B 507, 134 (1997).

2. A. Ringwald and F. Schrempp, Phys. Lett. B 438, 217 (1998).

3. H1 Collab. (C. Adloff et al.), Eur. Phys. J. C 25, 495 (2002).

4. ZEUS Collab. (S. Chekanov et al.), Eur. Phys. J. C 34, 255 (2004).

5. A. Ringwald and F. Schrempp, Comp. Phys. Commun. 132, 267 (2000).

6. G. Marchesini et al., Comp. Phys. Commun. 67, 465 (1992).

7. M.-M. Xu, et al., in preparation.

8. A. Białas and R. Peschanski, Nucl. Phys. B 273, 703 (1986).

9. W. Ochs, Phys. Lett. B 347, 101 (1990).

10. Y.-F. Wu and L.-S. Liu, Phys. Rev. Lett. 21, 3187 (1993).

11. Y.-F. Wu and L.-S. Liu, Sci. China, Ser. A 38, 435 (1995).

12. W. Ochs, Z. Phys. C 50, 339 (1991).

13. L.-S. Liu, Y. Zhang and Y. Deng, Z. Phys. C 73, 535 (1997).

14. W.-B. Deng et al., Entropy and equilibrium property of QCD-instanton induced final state in deep-inelastic scattering, to be published.

15. L.-S. Liu, G. Chen and J.-H. Fu, Phys. Rev. D 63, 054002 (2001).

16. K.-S. Zhang, G. Chen and L.-S. Liu, Int. J. Mod. Phys. A 17, 4543 (2002).

17. K.-S. Zhang, J.-W. Yin and G. Chen et al., HEPENP 26, 158 (2002) (in Chinese).

18. G. Chen, K.-S. Zhang and L.-S. Liu, Sci. China, Ser. G 46, 268 (2003). 